Center

for

Economic Research

No. $2000-52$

STRATEGIC TECHNOLOGY ADOPTION

TAKING INTO ACCOUNT FUTURE

TECHNOLOGICAL IMPROVEMENTS: A REAL

OPTIONS APPROACH

By Kuno J.M. Huisman and Peter M. Kort

May 2000

ISSN 0924-7815 


\title{
Strategic Technology Adoption Taking Into Account Future Technological Improvements: A Real Options
} Approach*

\author{
Kuno J. M. Huisman and Peter M. KorT ${ }^{\dagger}$ \\ Department of Econometrics and CentER, Tilburg University, \\ PO box 90153, 5000 LE TILBURG, The Netherlands
}

This paper studies a dynamic duopoly in which firms compete in the adoption of new technologies. The innovation process is exogenous to the firms. It is assumed that there are two technologies. Technology 1 already exists at time zero and can be adopted any time at a given one-time cost. This is standard and leads to a preemption game. What is new in the paper is the presence of technology 2 that becomes available for adoption at some time in the future. Technology 2 is superior to technology 1 but firms cannot adopt it if they have adopted technology 1 before technology 2 arrives.

The motivation to analyze a setup like this is that it provides a framework where firms take into account technological progress in making their investment decisions. Clearly this topic is important and also the results show that the addition of a superior technology appearing somewhere in the future can have substantial implications for the optimal investment decision. For example, adding technology 2 to the model turns the preemption game into a war of attrition in case of a high arrival probability. Another main result is that revenue uncertainty induces adoption of modern technologies.

Keywords: Technological uncertainty, Adoption, Preemption, War of attrition, Real options JEL classification: G31; L13; O31; O32

\footnotetext{
* The authors thank Dolf Talman for his constructive comments.

${ }^{\dagger}$ Corresponding author. Tel: +31-13-4662062; fax: +31-13-4663280; e-mail: kort@kub.nl
} 


\section{INTRODUCTION}

This paper is a contribution to the areas of technology adoption and investment under uncertainty. The aim is to determine the effects of technological progress on current investment decisions.

Concerning the area of technology adoption, the first paper that is important for our work is the seminal contribution by Fudenberg and Tirole (1985). They study a scenario of a duopoly with identical firms that both have the option to upgrade their technology. To do so they have to pay a sunk cost, while it holds that this sunk cost decreases over time. Thus the later a firm acquires this technology the less it costs. It follows that the optimal investment decision faces a trade off in the sense that investing soon implies that the firm can produce more efficiently from an early point in time onwards, but on the other hand large sunk costs have to be paid. Fudenberg and Tirole (1985) consider endogenous firm roles, so that it is not determined beforehand which firm will be the first investor. They show that the outcome is either a preemption equilibrium with dispersed investment timings and rent equalization, or a joint adoption equilibrium.

Recent extensions of the Fudenberg-Tirole framework include Stenbacka and Tombak (1994) (see also Götz (2000)), in which an uncertain time period is introduced, that ranges from the acquisition time to the time that the technology is successfully implemented. Another recent extension can be found in Hoppe (2000), where uncertainty concerning the performance of the new technology is introduced. Once the technology is acquired, the true value of the technology is revealed, which is observed by the rival firm. In this way, the firm that invests second free-rides on the risk the first firm has taken by investing in a technology 
of unknown value, which may lead to second mover advantages.

In Dixit and Pindyck (1996, Subsection 9.3) the Fudenberg-Tirole framework is combined with the theory of investment under uncertainty (real options), by imposing that the firm's profit flow is no longer deterministic, but satisfies a Brownian motion process instead. Nielsen (1999) shows that in this model competition on the output market decreases the option value of waiting and therefore duopolistic firms will invest earlier than monopolistic firms. Unlike Fudenberg and Tirole (1985), both Nielsen (1999) and Dixit and Pindyck (1996, Subsection 9.3) consider a new market model, which means that the firm starts being active after adopting the technology. In a new market model only the preemption equilibrium holds, meaning that the joint adoption equilibrium no longer applies. In Huisman and Kort (1999a) this new market model is extended by imposing that firms are already active on the market before the technology is upgraded. Consequently, the joint adoption equilibrium is restored. In Huisman and Kort (1999a) scenarios are identified in which either the preemption equilibrium or the joint adoption equilibrium prevails.

The research presented in this paper is motivated by the fact that present investment decisions are influenced by technological progress. A firm that invests today faces the risk that a much better technology becomes available tomorrow. The fact that this can happen provides an incentive to delay the investment. To include this kind of mechanism, the paper extends the model of Dixit and Pindyck (1996, Subsection 9.3) by incorporating an additional technology that becomes available at an unknown point of time in the future. This means that our model contains two different technologies that can be adopted, i.e. the technology that is currently available, and the most efficient technology that becomes available at a future point of time. At the moment the firm invests, it enters the market, so, like Dixit and 
Pindyck (1996, Subsection 9.3) we are considering a new market model. In this framework the possible invention of a more efficient technology raises the option value of waiting to invest in the current technology, but on the other hand the presence of a competitor may induce the firm to invest quickly, and thus forget about future technological progress.

Our results show that taking into account that a superior technology will be invented in the future can have substantial implications for the firm's investment decision. Compared to the case where technological progress is not included (Dixit and Pindyck (1996, Subsection 9.3)), taking into account the possible occurrence of a new technology could turn a preemption game into a war of attrition, which is a game where the second mover gets the highest payoff. This could happen when the first mover invests in the current technology, while the second mover waits for the new technology to arrive and invests then in it, and can be explained as follows. Compared to the strategy of its competitor, the benefits of the first investor are the monopoly profits gained during the period that starts at the moment of investment by the first investor and lasts until the moment that the second mover invests. However, these monopoly profits can be more than offset by the efficiency gain the second investor enjoys due to producing with a more efficient technology, which takes place after both firms have invested.

From the theory of real options it is known that the option value of waiting with investment increases with revenue uncertainty. For our model this implies that increased uncertainty delays adoption of the current technology, so that the probability that the new technology is invented before the investment in the current technology has taken place increases. This leads to the conclusion that increased revenue uncertainty induces a higher probability that the new technology will be adopted instead of the current technology. Hence, uncertainty 
raises the technological level within firms! Another result that is worth mentioning here is, that in a faster growing market a firm is more inclined to wait for a more efficient technology to arrive.

The organization of the paper is as follows. The model is presented in Section 2. After some preliminary analysis in Section 3, the outcome of the game for different probabilities concerning the future appearance of the new technology is presented in Section 4. Section 5 collects the economic implications.

\section{MODEL}

We consider two identical, risk neutral and value maximizing firms that can make an investment expenditure $I(>0)$ to become active on a market. We denote the firms by $i$ and $j$, with $i, j \in\{1,2\}$ and $i \neq j$. The firms discount future profits at rate $r(>0)$. At the beginning of the game, entering the market means producing with the existing technology 1. However, the decision to invest in technology 1 will be influenced by technological progress. Adopting technology 1 would have been a bad decision if a little later a much better technology becomes available. In our model technological progress is included as follows. At the stochastic time $T(>0)$ a new and better technology 2 becomes available for the firms. Time $T$ is distributed according to an exponential distribution with mean $\frac{1}{\lambda}(>0)$, so that the arrival of technology 2 follows a Poisson process with parameter $\lambda$.

Alternatively, in the one decision maker model in Grenadier and Weiss (1997), technological progress was modelled by assuming that it follows a Brownian motion process. At the moment that this process reaches a prespecified level, a new technology becomes available for adoption. By observing the difference between the current level of the Brownian motion 
process and this prespecified level, the firm has an idea about how long it still will last until the new technology arrives. In our model the arrival of a new technology follows a Poisson process so that at every point in time the probability that a new technology arrives is the same. Hence, our approach implicitly assumes that the firms have no information about the progress made within the research and development process of the technology. A disadvantage of the Grenadier and Weiss approach is that a Brownian motion process can be decreasing over time which leads to the strange observation that technological progress decreases as time passes.

We assume that firms can invest only once and that the investment costs of both technologies are equal. Concerning the profit flow it is assumed that it is stochastically evolving over time according to a Brownian motion process. We apply a specification frequently used in Dixit and Pindyck (1996). The profit flow of firm $i$ at time $t(\geq 0)$ equals

$$
\pi_{i}(t)=Y(t) D_{N_{i} N_{j}}
$$

where $N_{k}$ denotes the technology that firm $k(\in\{i, j\})$ is using. Hence, $N_{k} \in\{0,1,2\}$, where 0 means that the firm is not active. $Y(t)$ follows a geometric Brownian motion process

$$
\begin{aligned}
d Y(t) & =\mu Y(t) d t+\sigma Y(t) d \omega(t), \\
Y(0) & =y
\end{aligned}
$$

where $\mu(\in(0, r))$ is the drift parameter, $\sigma(>0)$ is the volatility parameter, $y(>0)$ is the starting value, and $d \omega(t)$ is an increment of a Wiener process. Thus $d \omega(t)$ is distributed according to a normal distribution with mean zero and variance $d t$. In the remainder of the paper we omit the time dependence of $Y(t)$ whenever there is no confusion possible.

We make the following assumptions on the $D$ 's. First, a firm makes the highest amount of profits with a given technology if the other firm is not active (monopoly). It also holds that, 
given its own technology, profits are lowest when the other firm is a strong competitor, thus producing with the efficient technology 2. Second, given the technology of the competitor, the firm's profits are higher when it produces with the modern technology 2. In this way the following inequalities are obtained:

$$
\begin{gathered}
D_{20}>D_{21}>D_{22} \\
\vee \quad \vee \quad \vee \\
D_{10}>D_{11}>D_{12}
\end{gathered}
$$

Finally, since it is a new market model, firms do not earn anything as long as they have not adopted a technology. This implies that, for $N_{k} \in\{0,1,2\}$ :

$$
D_{0 N_{k}}=0 .
$$

\section{Preliminary Analysis}

Our aim is to establish the analysis for purely identical firms. This means that it is not decided beforehand which firm will be leader or follower, i.e. which firm will be the first to invest. The following section presents equilibria for this scenario. However, before being able to do so, we first have to determine the payoffs in case the firm roles are fixed beforehand, which is done in this section. Here one firm gets the leader role and invests first. The other firm is the follower, which has the choice to invest at the same time as the leader or later. In Subsection 3.1 we consider the case where both technologies are already available, thus where $t \geq T$. In Subsection 3.2 technology 2 has not been invented yet.

\subsection{AnAlysis FOR $t \geq T$}

Three cases are possible when the second technology is already available. First, we consider the case where no firm has invested before time $T$, followed by the case where only the leader 
has invested before T. Finally, we give the payoff for the case that both firms have already invested before $T$.

\subsubsection{No Investment Before Time $T$}

Since $t \geq T$, technology 2 is already available for adoption. This technology is more efficient than technology 1, and therefore the firms will never invest in technology 1 . Hence, a game arises in which both firms consider entering a market by investing in one available technology, where the profit flow evolves stochastically over time. In fact, such a game is considered in Dixit and Pindyck (1996, Chapter 9) (see also Huisman and Kort (1999a)). In Huisman and Kort (1999a) it is shown that the expected value for each firm equals the follower value:

where

$$
\Phi_{22}(Y)= \begin{cases}A_{22} Y^{\beta_{1}} & \text { if } Y<Y_{22}^{F} \\ \frac{Y D_{22}}{r-\mu}-I & \text { if } Y \geq Y_{22}^{F}\end{cases}
$$

$$
\begin{aligned}
Y_{22}^{F} & =\frac{\beta_{1}}{\beta_{1}-1} \frac{(r-\mu) I}{D_{22}}, \\
A_{22} & =\left(Y_{22}^{F}\right)^{-\beta_{1}}\left(\frac{Y_{22}^{F} D_{22}}{r-\mu}-I\right), \\
\beta_{1} & =\frac{1}{2}-\frac{\mu}{\sigma^{2}}+\sqrt{\left(\frac{\mu}{\sigma^{2}}-\frac{1}{2}\right)^{2}+\frac{2 r}{\sigma^{2}}} .
\end{aligned}
$$

\subsubsection{One Investment Before Time $T$}

Here the leader has already invested in technology 1. Now the problem of the follower is in fact equal to that of a monopolist that considers entering a market where the profit flow equals $Y D_{21}$. From the analysis of this standard investment problem (see, e.g., Dixit and 
Pindyck (1996)) it is obtained that the value of the follower equals

where

$$
\Phi_{12}(Y)= \begin{cases}A_{12} Y^{\beta_{1}} & \text { if } Y<Y_{12}^{F}, \\ \frac{Y D_{21}}{r-\mu}-I & \text { if } Y \geq Y_{12}^{F},\end{cases}
$$

$$
\begin{aligned}
Y_{12}^{F} & =\frac{\beta_{1}}{\beta_{1}-1} \frac{(r-\mu) I}{D_{21}}, \\
A_{12} & =\left(Y_{12}^{F}\right)^{-\beta_{1}}\left(\frac{Y_{12}^{F} D_{21}}{r-\mu}-I\right) .
\end{aligned}
$$

The value of the leader follows automatically:

$$
\Lambda_{12}(Y)= \begin{cases}\frac{Y D_{10}}{r-\mu}+B_{12} Y^{\beta_{1}} & \text { if } Y<Y_{12}^{F}, \\ \frac{Y D_{12}}{r-\mu} & \text { if } Y \geq Y_{12}^{F} .\end{cases}
$$

When $Y<Y_{12}^{F}$ the profit flow is too low for the follower to invest. Therefore the leader enjoys monopoly profits. If the leader receives these forever, the leader's total profits would equal $\frac{Y D_{10}}{r-\mu}$. But it has to be taken into account that in the future $Y$ could reach $Y_{12}^{F}$ at a certain point of time. Then the follower will enter the market so that the leader's monopoly profits will be reduced. The term $B_{12} Y^{\beta_{1}}$ is the correction factor that incorporates this reduction into the firm's payoff for $Y<Y_{12}^{F}$. Therefore, the constant $B_{12}$ is negative and, due to the fact that the leader's value function is continuous at $Y_{12}^{F}$, it can be derived that

$$
B_{12}=\left(Y_{12}^{F}\right)^{1-\beta_{1}} \frac{D_{12}-D_{10}}{r-\mu}
$$

\subsubsection{Two Investments Before Time $T$}

The implication is that both firms have already invested in technology 1 . Therefore, the value of each firm equals

$$
\frac{Y D_{11}}{r-\mu}
$$




\subsection{AnAlysis FOR $t<T$}

First, the follower's problem is analyzed, followed by the problem of the leader. Then we consider the joint mover payoff, and finally we determine the expected payoff in case both firms wait for technology 2.

\subsubsection{FOLLOWER}

First, we determine the follower's value if the follower waits for technology 2 , while the leader has already invested in technology 1 . Then we consider the case where the follower can also invest in technology 1 , and determine the scenario under which investing in technology 1 can be optimal for the follower.

\section{Follower WAiting for Technology 2}

The value of the follower is denoted by $F_{12}(Y)$, and must satisfy the following Bellman equation

$$
r F_{12}(Y)=\lim _{d t \downarrow 0} \frac{1}{d t} E\left[d F_{12}(Y)\right]
$$

Itô's lemma tells us that (for the definition of $\Phi_{12}(Y)$ see $(10)$ ):

$$
\begin{aligned}
E\left[d F_{12}(Y)\right]= & (1-\lambda d t)\left(\frac{\partial F_{12}(Y)}{\partial Y} \mu Y d t+\frac{\partial^{2} F_{12}(Y)}{\partial Y^{2}} \frac{1}{2} \sigma^{2} Y^{2} d t\right) \\
& +\lambda d t\left(\Phi_{12}(Y)-F_{12}(Y)\right)+o(d t) .
\end{aligned}
$$

Substitution of (17) in (16) gives

$$
\frac{\partial F_{12}(Y)}{\partial Y} \mu Y+\frac{\partial^{2} F_{12}(Y)}{\partial Y^{2}} \frac{1}{2} \sigma^{2} Y^{2}-(r+\lambda) F_{12}(Y)+\lambda \Phi_{12}(Y)=0 .
$$


Using the two possible expressions for $\Phi_{12}(Y)$ (see (10)), the solution of (18) equals

$$
F_{12}(Y)= \begin{cases}\gamma_{1} Y^{\beta_{1}^{*}}+A_{12} Y^{\beta_{1}} & \text { if } Y<Y_{12}^{F}, \\ \gamma_{2} Y^{\beta_{2}^{*}}+\frac{\lambda}{r+\lambda-\mu} \frac{Y D_{21}}{r-\mu}-\frac{\lambda I}{r+\lambda} & \text { if } Y \geq Y_{12}^{F},\end{cases}
$$

where $\beta_{1}^{*}\left(\beta_{2}^{*}\right)$ is the positive (negative) solution of

$$
\frac{1}{2} \sigma^{2} \beta^{*}\left(\beta^{*}-1\right)+\mu \beta^{*}-(r+\lambda)=0
$$

Expressions for $\gamma_{1}$ and $\gamma_{2}$ are found by solving the continuity and the differentiability conditions for $F_{12}$ at $Y=Y_{12}^{F}$. This is done in Appendix A.1. It turns out that $\gamma_{1}<0$ and $\gamma_{2}>0$. In equation (19) we see that for $Y<Y_{12}^{F}$ the expected value of the follower consists of two parts. The second part equals the value of the option to adopt technology 2 (cf. equation (10)). The first part is a (negative) correction term, due to the fact that technology 2 is not available yet. Whenever $Y$ is above the threshold $Y_{12}^{F}$ the follower is going to adopt technology 2 at the moment that it becomes available. This last observation explains the last two terms of equation (19). The second term equals the expected present value of the profit flows generated from time $T$ onwards:

$$
\begin{aligned}
& E\left[\exp (-r T) \frac{Y(T) D_{21}}{r-\mu} \mid Y(0)=Y\right] \\
= & \frac{D_{21}}{r-\mu} E[\exp (-r T) Y(T) \mid Y(0)=Y] \\
= & \frac{D_{21}}{r-\mu} \int_{t=0}^{\infty} \lambda \exp (-\lambda t) \exp (-r t) E[Y(t) \mid Y(0)=Y] d t \\
= & \frac{D_{21}}{r-\mu} \int_{t=0}^{\infty} \lambda \exp (-\lambda t) \exp (-r t) Y \exp (\mu t) d t \\
= & \frac{\lambda}{r+\lambda-\mu} \frac{Y D_{21}}{r-\mu} .
\end{aligned}
$$


The third term is the expected present value of the investment cost that firm has to pay at time $T$ in order to adopt technology 2 :

$$
\begin{aligned}
& E[I \exp (-r T)] \\
= & I \int_{t=0}^{\infty} \lambda \exp (-\lambda t) \exp (-r t) d t \\
= & \frac{\lambda}{r+\lambda} I .
\end{aligned}
$$

Please note the difference between equations (21) and (22), i.e. the factors $\frac{\lambda}{r+\lambda-\mu}$ and $\frac{\lambda}{r+\lambda}$. In equation (21) the $\mu$ is subtracted from the denominator, in order to take into account the expected increase of $Y$.

If currently it holds that $Y(t) \geq Y_{12}^{F}$, it can still be the case that $Y$ lies below the threshold $Y_{12}^{F}$ at the time that the second technology arrives. Therefore, the correction term $\gamma_{2} Y^{\beta_{2}^{*}}$, is added to the follower's value. This correction term is positive, since it reflects the fact that the firm is not committed to make an investment. Undertaking the investment would be suboptimal when $Y$ is below $Y_{12}^{F}$ at the moment the new technology is invented. Thus $\gamma_{2} Y^{\beta_{2}^{*}}$ values flexibility. Notice that this correction factor vanishes when $Y$ goes to infinity. This for the reason that the probability that $Y(T)$ is below $Y_{12}^{F}$ goes to zero when $Y$ goes to infinity.

\section{Follower Considering Technology 1 to Be Interesting}

When $Y$ increases, the opportunity costs of waiting rise. This could imply that, given that the probability that a more efficient technology is invented soon is sufficiently low, the follower is going to adopt technology 1 for large values of $Y$. Therefore, intuition suggests that, in case of $\lambda$ sufficiently low, there exists a threshold $Y_{11}^{F}$ such that the follower will wait 
with investing if $Y<Y_{11}^{F}$ and for $Y \geq Y_{11}^{F}$ the follower will adopt technology 1 . Then the value of the follower is denoted by $F_{11}(Y)$ and equal to

$$
F_{11}(Y)= \begin{cases}\delta_{1} Y^{\beta_{1}^{*}}+A_{12} Y^{\beta_{1}} & \text { if } Y \in\left[0, Y_{12}^{F}\right), \\ \delta_{2} Y^{\beta_{1}^{*}}+\delta_{3} Y^{\beta_{2}^{*}}+\frac{\lambda}{r+\lambda-\mu} \frac{Y D_{21}}{r-\mu}-\frac{\lambda I}{r+\lambda} & \text { if } Y \in\left[Y_{12}^{F}, Y_{11}^{F}\right), \\ \frac{Y D_{11}}{r-\mu}-I & \text { if } Y \in\left[Y_{11}^{F}, \infty\right) .\end{cases}
$$

Equation (23) is derived by solving the follower's optimal stopping problem (see Appendix A.1). Solving the continuity and differentiability conditions for $F_{11}$ at $Y=Y_{12}^{F}$ and the value matching and smooth pasting condition for $F_{11}$ at $Y=Y_{11}^{F}$ gives expressions for the constants $\delta_{1}, \delta_{2}$ and $\delta_{3}$ (which can be found in Appendix A.1).

The term $\delta_{1} Y^{\beta_{1}^{*}}$ consists of two parts. The first part, $\left(\delta_{1}-\delta_{2}\right) Y^{\beta_{1}^{*}}$, is a correction term in the same fashion as $\gamma_{1} Y^{\beta_{1}^{*}}$ and the second part, $\delta_{2} Y^{\beta_{1}^{*}}$, is the value of the option to adopt technology 1. It turns out that the correction factor always dominates the option value and therefore $\delta_{1}<0$. The interpretation of $A_{12} Y^{\beta_{1}}$ is equal to the interpretation of the same factor in equation (19). The term $\delta_{2} Y^{\beta_{1}^{*}}$ equals the option value of adopting technology 1 , which implies that $\delta_{2}>0$. The correction factor $\delta_{3} Y^{\beta_{2}^{*}}$ is exactly equal to $\gamma_{2} Y^{\beta_{2}^{*}}$, thus $\delta_{3}>0$. Lemma 2 in Appendix B states the signs of the constants.

The following equation implicitly determines $Y_{11}^{F}$ (cf. Appendix A.1):

$$
\left(\beta_{1}^{*}-\beta_{2}^{*}\right) \delta_{3}\left(Y_{11}^{F}\right)^{\beta_{2}^{*}}+\frac{\left(\beta_{1}^{*}-1\right) \lambda Y_{11}^{F} D_{21}}{(r+\lambda-\mu)(r-\mu)}-\frac{\left(\beta_{1}^{*}-1\right) Y_{11}^{F} D_{11}}{r-\mu}+\frac{r \beta_{1}^{*} I}{r+\lambda}=0 .
$$

\section{Proposition 1}

The threshold $Y_{11}^{F}$ has the following properties:

(i) $Y_{11}^{F}$ only exists if $\lambda<\lambda_{1}^{*}$, where

$$
\lambda_{1}^{*}=\frac{(r-\mu) D_{11}}{D_{21}-D_{11}}
$$


(ii) $Y_{11}^{F}$ approaches the follower's threshold for adopting technology 1 in a model without technology 2 (see Dixit and Pindyck (1996, Section 9.3)) if $\lambda$ approaches zero, i.e.

$$
\lim _{\lambda \downarrow 0} Y_{11}^{F}(\lambda)=\frac{\beta_{1}}{\beta_{1}-1} \frac{(r-\mu) I}{D_{11}} .
$$

(iii) $Y_{11}^{F}$ approaches infinity if $\lambda$ approaches $\lambda_{1}^{*}$.

A proof of Proposition 1 can be found in Appendix B. It is intuitively clear that the threshold $Y_{11}^{F}$ is rising with $\lambda$, but due to the complexity of expression (24) it was impossible to find an analytical proof for this statement. A larger $\lambda$ implies that technology 2 is expected to arrive sooner and therefore it is in the follower's interest to postpone the adoption of technology 1 . Hence, the threshold for adopting technology 1 will be set higher.

The follower postpones the adoption of technology 1 forever when $Y_{11}^{F}$ approaches infinity. It is easy to verify that $\lim _{Y_{11}^{F} \rightarrow \infty} \delta_{1}=\gamma_{1}, \lim _{Y_{11}^{F} \rightarrow \infty} \delta_{2}=0$ and $\delta_{3}=\gamma_{2}$. This implies that equation (23) turns into equation (19) when $Y_{11}^{F}$ goes to infinity.

\subsubsection{LEADER}

Here we consider the case where the leader invests in technology 1 (for the case where the leader invests in technology 2, see Subsection 3.1). Two scenarios are analyzed. In the first scenario the follower only considers investing in technology 2 , while in the second scenario investing in technology 1 is an alternative for the follower.

\section{Follower Waiting For Technology 2}

When the follower waits for technology 2 , the value of the leader equals

$$
L_{12}(Y)=E\left[-I+\int_{t=0}^{T} Y(t) D_{10} \exp (-r t) d t+\exp (-r T) \Lambda_{12}(Y(T)) \mid Y(0)=Y\right] \text {. }
$$


This leads to the following expression for the leader curve (see (13), (14), and Appendix A.2)

$$
L_{12}(Y)= \begin{cases}\varepsilon_{1} Y^{\beta_{1}^{*}}+B_{12} Y^{\beta_{1}}+\frac{Y D_{10}}{r-\mu}-I & \text { if } Y<Y_{12}^{F}, \\ \varepsilon_{2} Y^{\beta_{2}^{*}}+\frac{Y D_{10}}{r+\lambda-\mu}+\frac{\lambda}{r+\lambda-\mu} \frac{Y D_{12}}{r-\mu}-I & \text { if } Y \geq Y_{12}^{F} .\end{cases}
$$

Expressions for $\varepsilon_{1}$ and $\varepsilon_{2}$ are derived by solving the continuity and differentiability conditions for $L_{12}$ at $Y=Y_{12}^{F}$, which is done in Appendix A.2. Lemma 4 in Appendix B states that $\varepsilon_{1}$ and $\varepsilon_{2}$ are both positive. The terms $\varepsilon_{1} Y^{\beta_{1}^{*}}$ and $\varepsilon_{2} Y^{\beta_{2}^{*}}$ correct for the fact that technology 2 has to arrive before the follower can adopt that technology and the leader's value becomes $\Lambda_{12}$. The longer it takes before technology 2 arrives, the longer the leader makes monopoly profits, i.e. the better for the leader. As in (13), $B_{12} Y^{\beta_{1}}$ stands for the option that $Y$ exceeds $Y_{12}^{F}$, so that the follower will adopt technology 2 , which ends the leader's monopoly profits. Consequently, as can be seen in (14), $B_{12}$ is negative. The value $\varepsilon_{2} Y^{\beta_{2}^{*}}$ equals the option that $Y$ falls below $Y_{12}^{F}$. This is good for the leader because if $Y<Y_{12}^{F}$ the follower will not invest so that the leader keeps on having monopoly profits. This explains why $\varepsilon_{2}$ is positive.

\section{Follower Considering Technology 1 to Be Interesting}

In this case the value of the leader is given by

$$
L_{11}(Y)= \begin{cases}\phi_{1} Y^{\beta_{1}^{*}}+B_{12} Y^{\beta_{1}}+\frac{Y D_{10}}{r-\mu}-I & \text { if } Y \in\left[0, Y_{12}^{F}\right), \\ \phi_{2} Y^{\beta_{1}^{*}}+\phi_{3} Y^{\beta_{2}^{*}}+\frac{Y D_{10}}{r+\lambda-\mu}+\frac{\lambda}{r+\lambda-\mu} \frac{Y D_{12}}{r-\mu}-I & \text { if } Y \in\left[Y_{12}^{F}, Y_{11}^{F}\right), \\ \frac{Y D_{11}}{r-\mu}-I & \text { if } Y \in\left[Y_{11}^{F}, \infty\right) .\end{cases}
$$

The derivation of equation (29) and expressions for $\phi_{1}, \phi_{2}$ and $\phi_{3}$ can be found in Appendix

A.2. The signs of $\phi_{1}$ and $\phi_{3}$ are equal to the signs of $\varepsilon_{1}$ and $\varepsilon_{2}$ in (28), respectively (see Lemma 5 in Appendix B).

The constant $\phi_{2}$ values the possibility that $Y$ rises above $Y_{11}^{F}$ before technology 2 arrives. On the one hand that event is good for the leader, since the follower adopts technology 1 
and not technology 2. On the other hand it is bad for the leader, because it no longer has a monopoly position. The following proposition states under which conditions $\phi_{2}$ is negative or positive, i.e. which argument dominates the other. The proof is given in appendix B.

\section{Proposition 2}

A sufficient condition for the constant $\phi_{2}$ to be non-positive is

$$
\frac{D_{21}}{D_{11}} \geq \frac{D_{12}-D_{10}}{D_{11}-D_{10}}
$$

If equation (30) does not hold, the sign of $\phi_{2}$ can go both ways.

Equation (30) states that the relative profit gain the follower can make by adopting technology 2 is larger than the relative profit loss that the leader faces when the follower adopts technology 2. Inequality (30) is most likely to hold when the leader is almost indifferent concerning the technology the follower switches to. In that case it is not good for the leader if the follower switches to 1 immediately rather than waiting for 2 . Consequently $\phi_{2}$ is negative which is confirmed by Proposition 2 .

\subsubsection{JoInt INVESTMENT}

The expected value of each firm if both firms adopt technology 1 together is given by

$$
M_{11}(Y)=\frac{Y D_{11}}{r-\mu}-I .
$$




\subsubsection{Waiting Curve}

The waiting curve (see also Huisman and Kort (1999b)) gives the expected value if both firms wait with investing until technology 2 arrives. The waiting curve equals

$$
\begin{aligned}
W(Y) & =E\left[\exp (-r T) \Phi_{22}(Y(T)) \mid Y(0)=Y\right] \\
& = \begin{cases}\eta_{1} Y^{\beta_{1}^{*}}+A_{22} Y^{\beta_{1}} & \text { if } Y<Y_{22}^{F}, \\
\eta_{2} Y^{\beta_{2}^{*}}+\frac{\lambda Y D_{22}}{(r+\lambda-\mu)(r-\mu)}-\frac{\lambda I}{r+\lambda} & \text { if } Y \geq Y_{22}^{F} .\end{cases}
\end{aligned}
$$

For a derivation we refer to Appendix A.3. There we also present expressions for $\eta_{1}$ and $\eta_{2}$. The constant $\eta_{1}$ is negative and the constant $\eta_{2}$ is positive. These constants have the same economic interpretations as $\gamma_{1}$ and $\gamma_{2}$, respectively.

\section{Proposition 3}

It always holds that $F_{12}(Y)>W(Y)$.

This proposition is proved in Appendix B and is a direct result of the new market assumption. The follower starts making profits after its investment and from the follower's point of view it is best that the leader adopts technology 1.

\section{EQUILIBRIA}

In this section firm roles are endogenous which means that it is not determined beforehand which firm will be the first investor. We describe the possible equilibria of the technology adoption game before the arrival of technology 2. It turns out that the type of the equilibria is completely determined by $\lambda$. In the following theorem we describe this relationship.

\section{THEOREM 1}

There are three regions for $\lambda$. 
(i) If $\lambda \in\left[0, \lambda_{2}^{*}\right)$ the equilibrium is of the preemption type.

(ii) If $\lambda \in\left[\lambda_{2}^{*}, \lambda_{3}^{*}\right)$ the equilibrium is of the attrition type.

(iii) If $\lambda \in\left[\lambda_{3}^{*}, \infty\right)$ both firms wait with investing until technology 2 arrives.

The critical $\lambda$ levels are equal to

$$
\begin{aligned}
& \lambda_{2}^{*}=\frac{(r-\mu) D_{10}}{D_{21}-D_{12}}, \\
& \lambda_{3}^{*}=\frac{(r-\mu) D_{10}}{D_{22}-D_{12}} .
\end{aligned}
$$

The first $\lambda$ region is split up into two $\lambda$ regions: $\left[0, \lambda_{1}^{*}\right)$ and $\left[\lambda_{1}^{*}, \lambda_{2}^{*}\right)$, where $\lambda_{1}^{*}$ is given by (25). Note that equation (30) ensures that $\lambda_{1}^{*} \leq \lambda_{2}^{*}$. In case equation (30) does not hold, the second region for $\lambda$ does not exist. In each of the following four subsections one of the regions for $\lambda$ is analyzed and the equilibria are characterized. In the remainder of this section Theorem 1 is implicitly proved. The propositions in this section are proved in Appendix B.

\subsection{CASE 1}

In the first case we have $\lambda \in\left[0, \lambda_{1}^{*}\right)$. From the analysis of the previous section we know that in this region the follower is going to adopt technology 1 for $Y$ large enough. This implies that in the equilibrium analysis the leader curve is given by equation (29), the follower curve by (23), the joint investment curve by (31), and the waiting curve by (32). The following proposition states that there exists a preemption threshold in this region.

\section{Proposition 4}

Let $\lambda \in\left[0, \lambda_{1}^{*}\right)$. Then there exists a unique $Y_{11}^{P} \in\left(0, Y_{11}^{F}\right)$ such that

$$
L_{11}\left(Y_{11}^{P}\right)=F_{11}\left(Y_{11}^{P}\right) \text {. }
$$


Define $T_{11}^{P}$ and $T_{11}^{F}$ as follows: $T_{11}^{P}=\inf \left(t \mid Y(t) \geq Y_{11}^{P}\right)$ and $T_{11}^{F}=\inf \left(t \mid Y(t) \geq Y_{11}^{F}\right)$. Propositions 3 and 4 imply that the leader curve exceeds the waiting curve for some $Y$. From Huisman and Kort (1999b) it follows that the equilibria of this game with waiting curve are equal to equilibria of the game without waiting curve. This means that in analyzing the game the future arrival of technology 2 can be ignored for the moment (of course, if, despite the low probability, technology 2 arrives before one of the firms has invested in technology 1 , the outcome must be reconsidered). Hence, a game must be considered where two firms have to determine their optimal timing concerning the investment in a given technology. This is in fact the game described in Dixit and Pindyck (1996, Chapter 9), see Huisman and Kort (1999a). Here we repeat the most important aspects.

\section{THEOREM 2}

Consider the game with $y \leq Y_{11}^{P}$. It holds that in equilibrium the leader adopts technology 1 at time $T_{11}^{P}$ and the follower adopts technology 1 at time $T_{11}^{F}$.

Of course, Theorem 2 is conditional on the fact that technology 2 does not arrive before time $T_{11}^{F}$. Further we should remark that if $Y_{11}^{P}<y<Y_{11}^{F}$ there exists a positive probability that the firms invest simultaneously at time 0 (cf. Huisman and Kort (1999a)). In equilibrium the expected value of each firm equals the follower value. Figure 1 graphically shows the curves in this case.

The investment opportunity is worthless for $Y$ equal to zero. Therefore, at $Y=0$ the leader $(L)$ and joint investment $(M)$ value equal minus the investment cost and the follower $(F)$ value equals zero. The further shape of the curves $L, F, M$ and $W$ can be derived from (23), (29), (31), (32), and (35). 


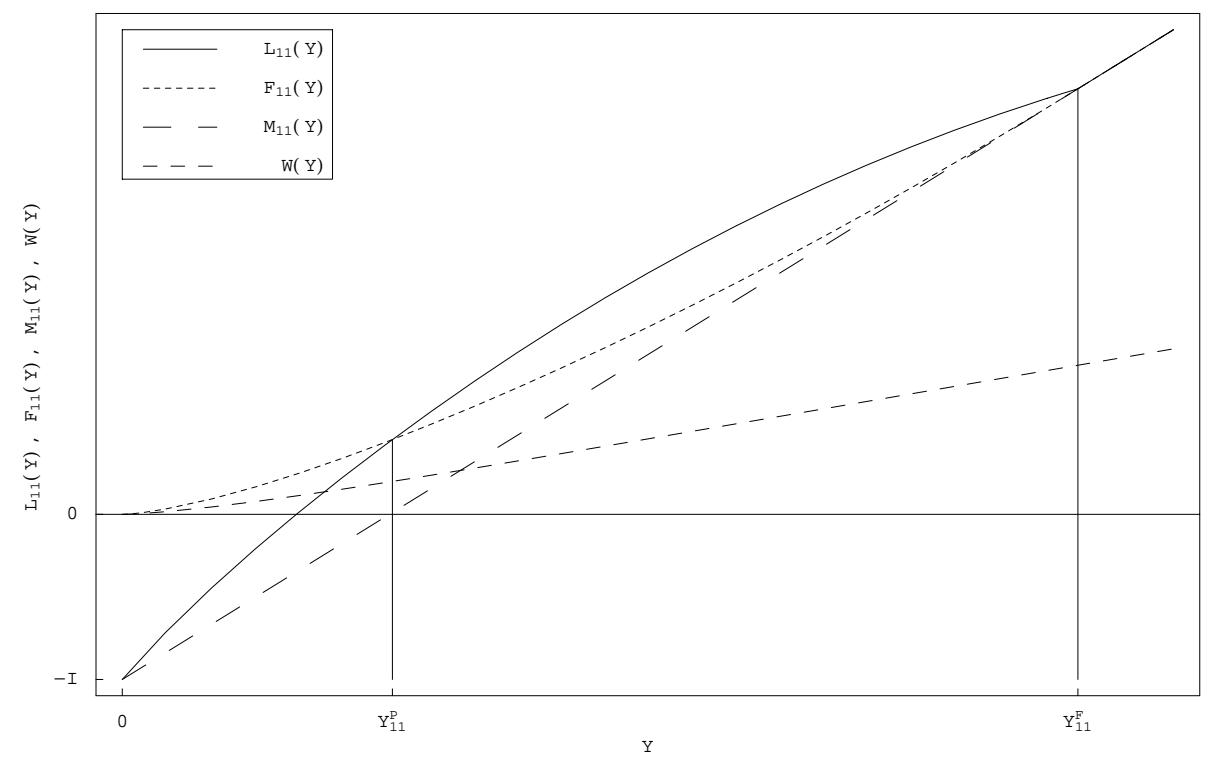

Figure 1. Case 1: $\lambda \in\left[0, \lambda_{1}^{*}\right)$.

With Figure 1 the preemption mechanism can be clearly explained. Consider the game with $Y(0) \leq Y_{11}^{P}$. Assume that both firms pass $Y_{11}^{P}$ without investing and the current value of $Y$, say $Y(t)$, exceeds $Y_{11}^{P}$. Then for one of the firms it is optimal to invest at time $t$, since the $L$-curve lies above the $F$-curve, implying that investing first gives a higher payoff than investing second. The other firm knows this and will try to preempt its competitor by investing at time $t-\epsilon$, since it knows that the other firm would like to be the first to invest at time $t$. But then the other firm will try to preempt at time $t-2 \epsilon$. It is clear that this process of preemption stops at $Y_{11}^{P}$, since for $Y<Y_{11}^{P}$ it holds that $F(Y)>L(Y)$ so that there are no incentives to invest first.

The following proposition gives an expression for the probability that technology 2 arrives after a certain threshold is hit. The proof of the proposition is given in Appendix B.

\section{Proposition 5}

Let $T_{S}=\inf (t \mid Y(t) \geq S)$. At time $t=0$ the probability that the geometric Brownian 
motion hits the threshold $S$ before the second technology arrives, i.e. $\operatorname{Pr}\left(T_{S}<T\right)$, is given by

where

$$
\operatorname{Pr}\left(T_{S}<T\right)= \begin{cases}\left(\frac{y}{S}\right)^{\widehat{\beta}_{1}} & \text { if } y<S \\ 1 & \text { if } y \geq S\end{cases}
$$

$$
\widehat{\beta}_{1}=\frac{1}{2}-\frac{\mu}{\sigma^{2}}+\sqrt{\left(\frac{\mu}{\sigma^{2}}-\frac{1}{2}\right)^{2}+\frac{2 \lambda}{\sigma^{2}}} .
$$

From Proposition 5 we derive that the probability that technology 1 is adopted by the leader (follower) decreases with $\lambda$. An increase of $\lambda$ leads to both a higher threshold and a higher $\widehat{\beta}_{1}$.

\subsection{CASE 2}

In the second case it holds that $\lambda \in\left[\lambda_{1}^{*}, \lambda_{2}^{*}\right)$. Here the probability that technology 2 arrives soon is that high that the follower is going to wait for technology 2. As in the previous case there exists a preemption threshold.

\section{Proposition 6}

Let $\lambda \in\left[\lambda_{1}^{*}, \lambda_{2}^{*}\right)$. Then there exists a unique $Y_{12}^{P} \in(0, \infty)$ such that

$$
L_{12}\left(Y_{12}^{P}\right)=F_{12}\left(Y_{12}^{P}\right) \text {. }
$$

We define $T_{12}^{P}$ in the same fashion as $T_{11}^{P}: T_{12}^{P}=\inf \left(t \mid Y(t) \geq Y_{12}^{P}\right)$. Furthermore we define $T_{12}^{F}=\inf \left(t \geq T \mid Y(t) \geq Y_{12}^{F}\right)$

\section{THEOREM 3}

In equilibrium the leader adopts technology 1 at time $T_{12}^{P}$ and the follower adopts technology 2 at time $T_{12}^{F}$. 
As above the leader's adoption of technology 1 is conditional on technology 2 not arriving before time $T_{12}^{P}$. If initially $Y$ is above $Y_{12}^{P}$ then with positive probability both firms adopt technology 1 at time 0 . The expected value of each firm equals the follower value. The curves for this case are plotted in Figure 2.

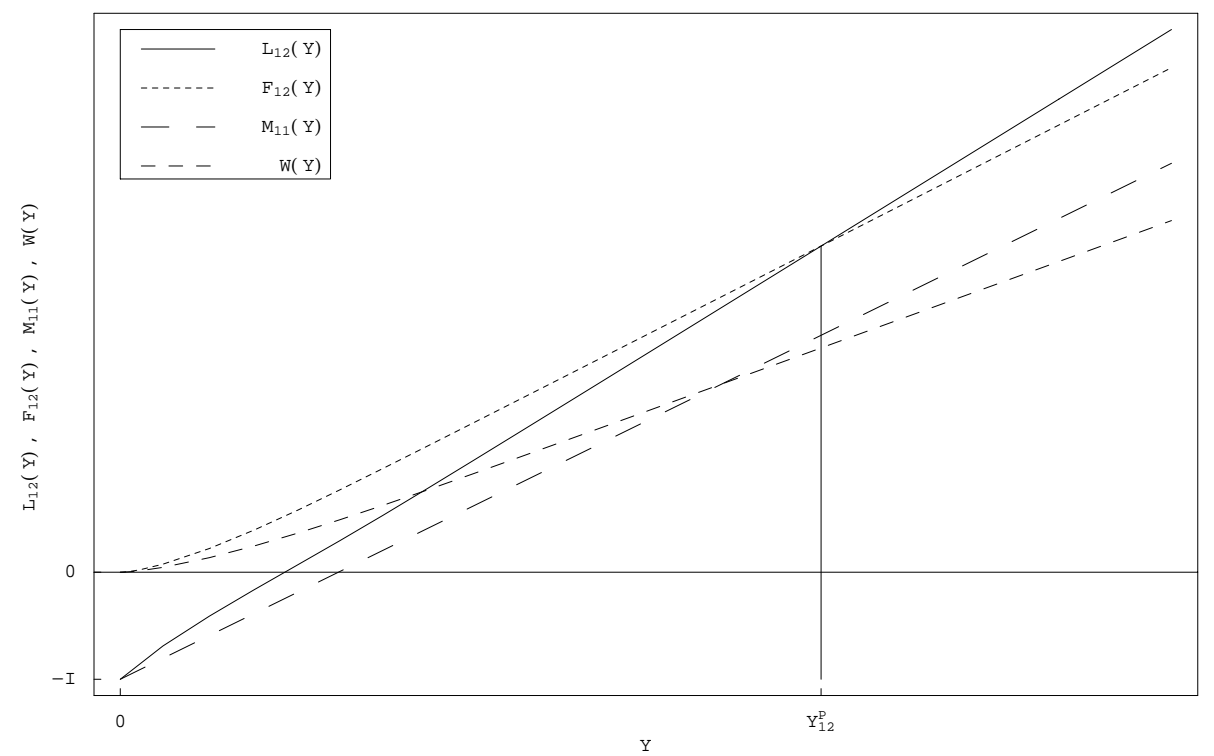

Figure 2. Case 2: $\lambda \in\left[\lambda_{1}^{*}, \lambda_{2}^{*}\right)$

From the fact that $Y_{12}^{P}$ is rising in $\lambda$ and Proposition 5 it can be concluded that the probability that the leader adopts technology 1 decreases with $\lambda$.

\subsection{CASE 3}

The third case is characterized by the fact that $\lambda \in\left[\lambda_{2}^{*}, \lambda_{3}^{*}\right)$. Here the probability that technology 2 arrives is even higher than in Case 2, where it was already high enough for the follower to wait for technology 2. This implies that also in this case the follower is going to wait for technology 2. In this region there does not exist a preemption threshold, i.e. the follower curve is situated above the leader curve for each $Y$. This implies that the game 
without waiting curve is an attrition game.

\section{Proposition 7}

Let $\lambda \in\left[\lambda_{2}^{*}, \lambda_{3}^{*}\right)$. Then there exists a unique $Y_{12}^{L} \in(0, \infty)$ such that

$$
L_{12}\left(Y_{12}^{L}\right)=W\left(Y_{12}^{L}\right)
$$

The following theorem describes the equilibrium conditional on technology 2 not arriving before time $T_{12}^{L}=\inf \left(t \mid Y(t) \geq Y_{12}^{L}\right)$.

\section{THEOREM 4}

In equilibrium the leader adopts technology 1 at time $T_{12}^{L}$ and the follower adopts technology 2 at time $T_{12}^{F}$.

The curves for the different payoffs in this game are depicted in Figure 3. The leader curve shows the expected payoff as function of $Y$ for a firm that invests in technology 1 immediately. This firm knows that its competitor will invest in technology 2 as soon as it becomes available and $Y>Y_{12}^{F}$. The leader has the advantage of monopoly profits until the time that the follower invests in technology 2, but the disadvantage of producing with a less efficient technology after this date. On the other hand the waiting curve shows the expected payoff if both firms wait for technology 2 to arrive. As long as the waiting curve lies above the leader curve, investing now in technology 1 is not a sensible option. Therefore, the attrition game starts at time $T_{12}^{L}$.

In the attrition game the follower curve is situated above the leader curve and the leader curve above the joint investment curve for all positive $Y$. This implies that there does not exist a symmetric equilibrium for this attrition game (cf. Hendricks et al. (1988)). There 


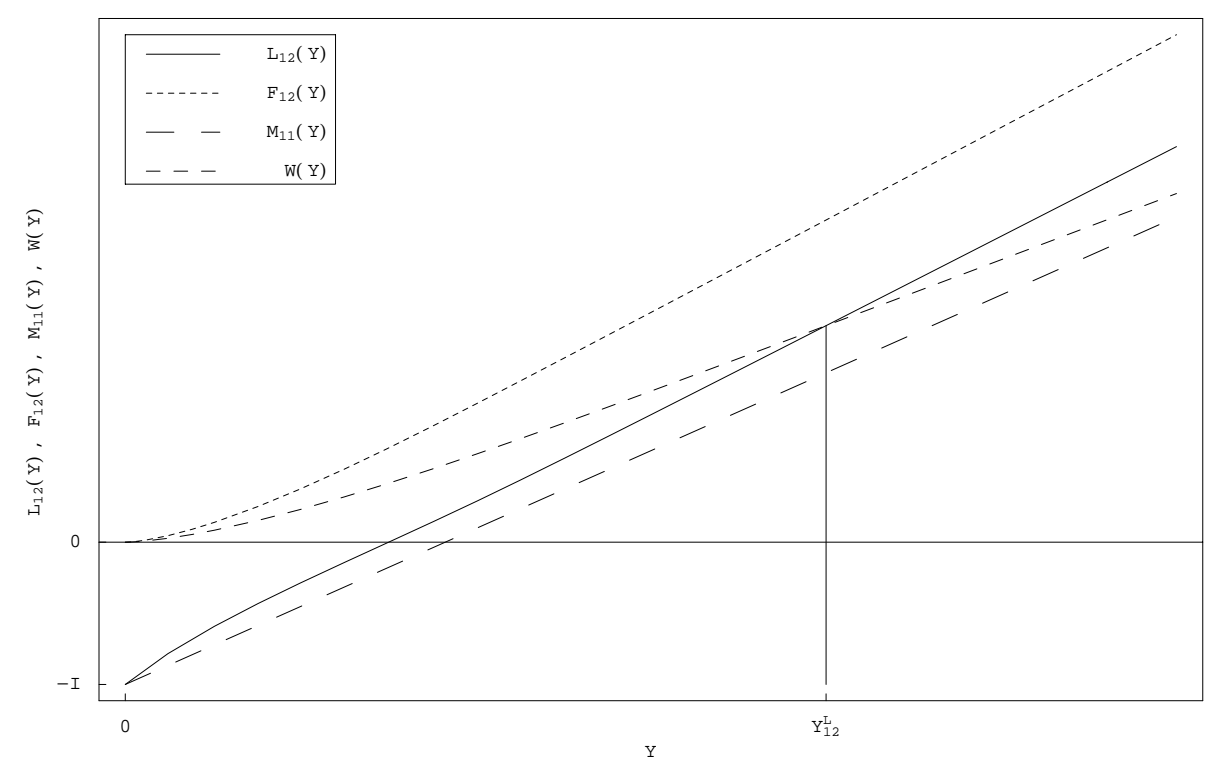

Figure 3. Case 3: $\lambda \in\left[\lambda_{2}^{*}, \lambda_{3}^{*}\right)$.

are two asymmetric equilibria, which are summarized in Theorem 4 (each firm can either be leader or follower). For simplicity reasons we assume that each equilibrium occurs with probability one half.

Proposition 5 together with the fact that $Y_{12}^{L}$ is increasing with $\lambda$ imply that the probability that the leader adopts technology 1 is decreasing with $\lambda$.

\subsection{CASE 4}

In the fourth case $\left(\lambda \in\left[\lambda_{3}^{*}, \infty\right)\right)$ the probability that technology 2 will be invented soon is that high that both firms wait with investing until technology 2 arrives. This is reflected by the fact that the waiting curve exceeds the leader curve for all $Y$ in this region. Figure 4 shows the curves in this case.

At the moment that technology 2 arrives, a game starts where both firms consider entering a market by investing in one available technology (the presence of technology 1 can be ignored since it is less efficient), while the profit flow follows a geometric Brownian motion process. 


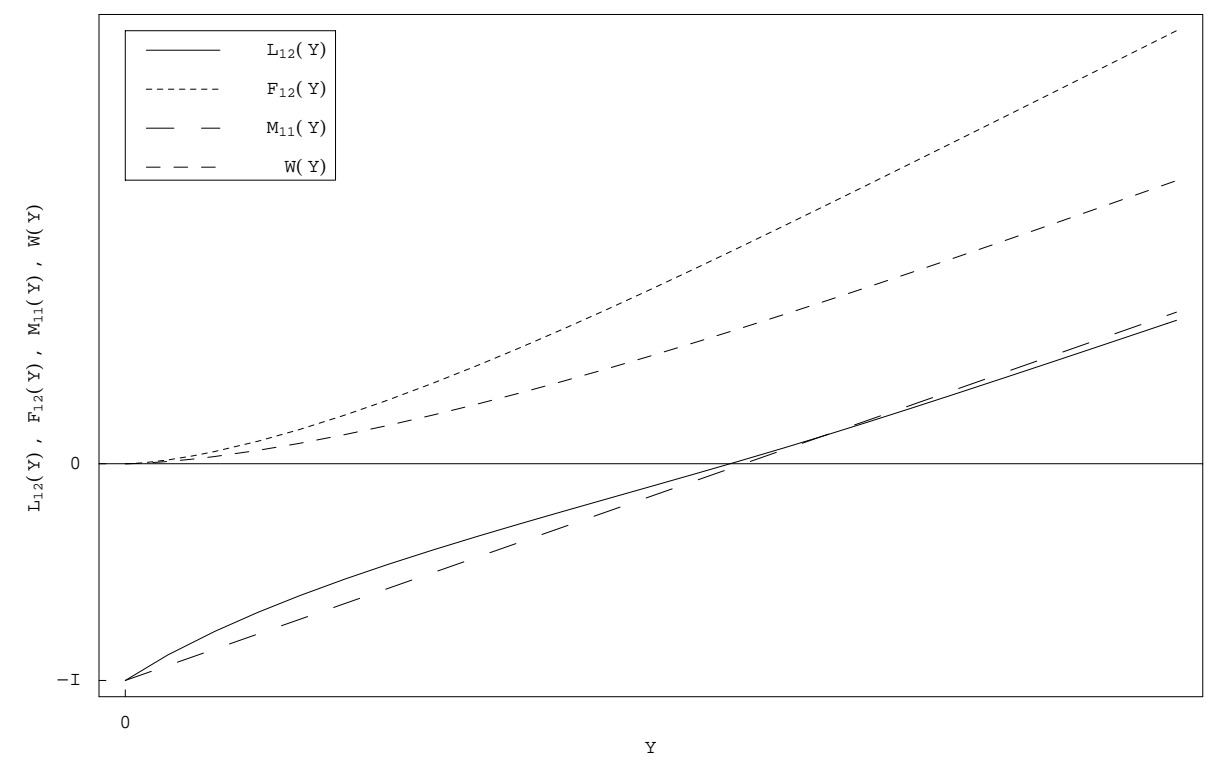

Figure 4. Case 4: $\lambda \in\left[\lambda_{3}^{*}, \infty\right)$.

Hence, like in Case 1, the framework of Dixit and Pindyck (1996, Chapter 9) again applies. The difference is that in Case 1 the Dixit and Pindyck game has to deal with investment in technology 1, while here the investment in technology 2 must be considered.

\section{ECONOMic AnALysis}

The Poisson parameter $\lambda$ is the key parameter for the results. Waiting for the new technology is better when the probability that this new technology becomes available soon, is high enough. If this probability is low enough both firms only consider when to invest in the current technology, while ignoring the new one. In this case the usual preemption game arises (see Fudenberg and Tirole (1985) and Dixit and Pindyck (1996, Chapter 9) for its stochastic counterpart).

If the probability that technology 2 becomes available soon is not too small, i.e. the Poisson parameter exceeds $\lambda_{1}^{*}$ (cf. (25)), then the game is still a preemption game, so that each firm tries to be the first investor. However, the firm that will invest second is better off 
by waiting for the new technology rather than investing in the current one.

If $\lambda$ is again a bit larger such that it exceeds $\lambda_{2}^{*}$ (see (33)), the preemption game turns into an attrition game. Like in the previous case, the first investor chooses the current technology and the second investor will wait for the new technology, but the difference is that the payoff of the second investor is higher here. Hence, neither firm would like to be the first investor, but if they both keep on waiting, their payoff will be even less than the payoff of the one that decides immediately to invest first. According to Hendricks et al. (1988) a unique asymmetric equilibrium exists where the adoption timings are dispersed.

If $\lambda$ exceeds $\lambda_{3}^{*}$, given by (34), then the probability that technology 2 arrives soon is that large that both firms will wait for this new technology. The possibility to invest in the current technology will be ignored.

It is clear that for $\lambda=0$ the model exactly equals the one treated in Dixit and Pindyck (1996, Chapter 9). Here there is no technological progress in the sense that the probability that a new technology will be invented is zero. Hence both firms only need to consider investing in the current technology, so that the problem boils down to the determination of the optimal point in time that a firm must enter a market with stochastic profit flow, while taking into account the behavior of an identical competitor. The resulting game is a preemption game, like the one where $\lambda$ is positive but below $\lambda_{1}^{*}$. It holds that $Y_{11}^{P}$ increases with $\lambda$ so that the possible occurrence of a new technology will delay investment in the current technology, which is intuitively plausible.

Comparing the case for $\lambda=0$ (model with one technology) with $\lambda \in\left(\lambda_{2}^{*}, \lambda_{3}^{*}\right)$ shows that taking into account the possible occurrence of a new technology could turn a preemption game into an attrition game. 
To learn more about the effects of the future availability of a more efficient technology on the optimal timing of investment, we also carry out comparative statics analysis on the other parameter values. Let us first consider the effect of revenue volatility which is measured by $\sigma$. The general prediction of the real options literature is that a higher level of uncertainty increases the threshold level and therefore will have a negative effect on investment. In our model an increased threshold level implies that the investment in technology 1 will be delayed. Therefore, the probability that technology 2 arrives before the investment is undertaken, increases. Hence, the conclusion is that increased revenue uncertainty induces a higher probability that the new technology will be adopted instead of the current technology.

Next, consider the expected growth of the market reflected by the parameter $\mu$. An increase of $\mu$ reduces the values of $\lambda_{i}^{*}$, with $i \in\{1,2,3\}$. In general this means that the probability increases that the firm will delay or totally refrain from investing in the current technology. The reason is that in case of a fast growing market the firm will exploit this growth as much as possible by using the more efficient new technology. The firm is more willing to wait for this technology to be invented.

The effect of the discount rate is completely opposite to the effect of the expected market growth rate. A higher discount rate implies that immediate profits are more important to the firm. Therefore the firm prefers investing in the current technology rather than waiting for the new one.

Finally, consider the effects of the several profit flows. First, notice that $\lambda_{1}^{*}$ increases with $D_{11}$ and decreases with $D_{21}$. This can be explained by the fact that the second investor is more willing to produce with the first technology if $D_{11}$ is large, while it likes to wait for the new technology to arrive if $D_{21}$ is large. 
Second, $\lambda_{2}^{*}$ increases with $D_{10}$ and $D_{12}$, while it decreases with $D_{21}$. This implies that $\lambda_{2}^{*}$ is larger if the payoff of the strategy "adopt technology 1 immediately" is higher relative to the payoff of the strategy "wait for technology 2 to arrive and adopt it then". Note that if the latter strategy gives the highest payoff, the game is an attrition game, which occurs for $\lambda \in\left(\lambda_{2}^{*}, \lambda_{3}^{*}\right)$

Third, $\lambda_{3}^{*}$ increases with $D_{10}$ and $D_{12}$, while it decreases with $D_{22}$. Hence, if a high profit is reached when both firms produce with the new technology, compared to the strategy "invest in technology 1 immediately and have some monopoly profits before technology 2 arrives", both firms will wait for the second technology to arrive. This in fact happens for $\lambda>\lambda_{3}^{*}$.

\section{APPENDIX}

\section{A Derivation of Value Functions}

\section{A.1 FOLLOWER}

\section{A.1.1 Follower Waiting for Technology 2}

Solving the continuity and differentiability conditions for $F_{12}(Y)$ at $Y=Y_{12}^{F}$ gives

$$
\begin{aligned}
& \gamma_{1}=\frac{\left(Y_{12}^{F}\right)^{-\beta_{1}^{*}} I\left(r(r-\mu) \beta_{2}^{*}+\left(r-\mu \beta_{1}\right) \lambda \beta_{2}^{*}-(r-\mu)(r+\lambda) \beta_{1}\right)}{(r+\lambda)(r+\lambda-\mu)\left(\beta_{1}-1\right)\left(\beta_{1}^{*}-\beta_{2}^{*}\right)}, \\
& \gamma_{2}=\frac{\left(Y_{12}^{F}\right)^{-\beta_{2}^{*}} I\left(r(r-\mu) \beta_{1}^{*}+\left(r-\mu \beta_{1}\right) \lambda \beta_{1}^{*}-(r-\mu)(r+\lambda) \beta_{1}\right)}{(r+\lambda)(r+\lambda-\mu)\left(\beta_{1}-1\right)\left(\beta_{1}^{*}-\beta_{2}^{*}\right)} .
\end{aligned}
$$

A direct result of Lemma 1 (see Appendix B) is that $\gamma_{1}<0$ and $\gamma_{2}>0$. 


\section{A.1.2 Follower Considering Technology 1 to Be Interesting}

The follower solves the optimal stopping problem, in which stopping means adopting technology 1. Therefore the expected value of the follower for $Y \geq Y_{11}^{F}$ equals

$$
F_{11}(Y)=\frac{Y D_{11}}{r-\mu}-I
$$

In the continuation region waiting is the optimal strategy and the following Bellman equation must be satisfied

$$
r F_{11}(Y)=\lim _{d t \downarrow 0} \frac{1}{d t} E\left[d F_{11}(Y)\right] .
$$

Expanding the right-hand-side of (43) with Itô's lemma and rewriting gives

$$
\frac{\partial F_{11}(Y)}{\partial Y} \mu Y+\frac{\partial^{2} F_{11}(Y)}{\partial Y^{2}} \frac{1}{2} \sigma^{2} Y^{2}-(r+\lambda) F_{11}(Y)+\lambda \Phi_{12}(Y)=0 .
$$

Using (10) and the boundary condition $F_{11}(0)=0$ gives

$$
F_{11}(Y)= \begin{cases}\delta_{1} Y^{\beta_{1}^{*}}+A_{12} Y^{\beta_{1}} & \text { if } Y<Y_{12}^{F}, \\ \delta_{2} Y^{\beta_{1}^{*}}+\delta_{3} Y^{\beta_{2}^{*}}+\frac{\lambda Y D_{21}}{(r+\lambda-\mu)(r-\mu)}-\frac{\lambda I}{r+\lambda} & \text { if } Y \geq Y_{12}^{F} .\end{cases}
$$

Combining (42) and (43) gives equation (23).

Expressions for $\delta_{1}, \delta_{2}, \delta_{3}$ and $Y_{11}^{F}$ are found by simultaneously solving the continuity and differentiability conditions for $F_{11}$ at $Y_{12}^{F}$ and the value matching and smooth pasting conditions for $F_{11}$ at $Y_{11}^{F}$. It turns out that it is not possible to get a closed form solution for $Y_{11}^{F}$. The threshold $Y_{11}^{F}$ is implicitly determined by equation (24). The constants are equal to

$$
\begin{aligned}
& \delta_{1}=\delta_{2}+\left(Y_{12}^{F}\right)^{\beta_{2}^{*}-\beta_{1}^{*}} \frac{\beta_{2}^{*} \gamma_{2}}{\beta_{1}^{*}}-\left(Y_{12}^{F}\right)^{1-\beta_{1}^{*}} \frac{D_{21}}{\beta_{1}^{*}(r+\lambda-\mu)}, \\
& \delta_{2}=\left(Y_{11}^{F}\right)^{\beta_{2}^{*}-\beta_{1}^{*}} \frac{\left(1-\beta_{2}^{*}\right) \gamma_{2}}{\beta_{1}^{*}-1}+\left(Y_{11}^{F}\right)^{-\beta_{1}^{*}} \frac{r I}{\left(\beta_{1}^{*}-1\right)(r+\lambda)} \\
& \delta_{3}=\gamma_{2} .
\end{aligned}
$$

Lemma 2 in Appendix B states that $\delta_{1}<0, \delta_{2}>0$, and $\delta_{3}>0$. 


\section{A.2 LEADER}

\section{A.2.1 Follower Waiting For Technology 2}

In order to derive an expression for equation (27), define

$$
h(Y)=E\left[\int_{t=0}^{T} Y(t) D_{10} \exp (-r t) d t+\exp (-r T) \Lambda_{12}(Y(T)) \mid Y(0)=Y\right] .
$$

Then $h(Y)$ must satisfy the following Bellman equation

$$
r h(Y)=Y D_{10}+\lim _{d t \downarrow 0} \frac{1}{d t} E[d h(Y)] .
$$

Applying Itô's lemma gives

$$
\begin{aligned}
E[d h(Y)]= & (1-\lambda d t)\left(\frac{\partial h(Y)}{\partial Y} \mu Y d t+\frac{\partial^{2} h(Y)}{\partial Y^{2}} \frac{1}{2} \sigma^{2} Y^{2} d t\right) \\
& +\lambda d t\left(\Lambda_{12}(Y)-h(Y)\right)+o(d t) .
\end{aligned}
$$

Substitution of (51) in (50) gives

$$
\frac{\partial h(Y)}{\partial Y} \mu Y+\frac{\partial^{2} h(Y)}{\partial Y^{2}} \frac{1}{2} \sigma^{2} Y^{2}-(r+\lambda) h(Y)+\lambda \Lambda_{12}(Y)+Y D_{10}=0 .
$$

Substitution of (13) in (52) and solving that differential equation gives

$$
h(Y)= \begin{cases}\varepsilon_{1} Y^{\beta_{1}^{*}}+\tau_{1} Y^{\beta_{2}^{*}}+B_{12} Y^{\beta_{1}}+\frac{Y D_{10}}{r-\mu} & \text { if } Y<Y_{12}^{F}, \\ \tau_{2} Y^{\beta_{1}^{*}}+\varepsilon_{2} Y^{\beta_{2}^{*}}+\frac{Y D_{10}}{r+\lambda-\mu}+\frac{\lambda}{r+\lambda-\mu} \frac{Y D_{12}}{r-\mu} & \text { if } Y \geq Y_{12}^{F} .\end{cases}
$$

The boundary condition at $Y=0$ and the condition that rules out speculative bubbles (see

Dixit and Pindyck (1996, p. 181)),

$$
\begin{aligned}
h(0) & =0, \\
\lim _{Y \rightarrow \infty} \frac{h(Y)}{Y} & =\frac{D_{10}}{r+\lambda-\mu}+\frac{\lambda}{r+\lambda-\mu} \frac{D_{12}}{r-\mu},
\end{aligned}
$$

imply that $\tau_{1}=0$ and $\tau_{2}=0$.

Solving the continuity and differentiability conditions for $L_{12}$ at $Y=Y_{12}^{F}$ gives 


$$
\begin{aligned}
& \varepsilon_{1}=\frac{\left(Y_{12}^{F}\right)^{1-\beta_{1}^{*}}\left((r-\mu)\left(\beta_{1}-\beta_{2}^{*}\right)+\lambda\left(\beta_{1}-1\right)\right)\left(D_{10}-D_{12}\right)}{(r+\lambda-\mu)(r-\mu)\left(\beta_{1}^{*}-\beta_{2}^{*}\right)} \\
& \varepsilon_{2}=\frac{\left(Y_{12}^{F}\right)^{1-\beta_{2}^{*}}\left((r-\mu)\left(\beta_{1}-\beta_{1}^{*}\right)+\lambda\left(\beta_{1}-1\right)\right)\left(D_{10}-D_{12}\right)}{(r+\lambda-\mu)(r-\mu)\left(\beta_{1}^{*}-\beta_{2}^{*}\right)} .
\end{aligned}
$$

According to Lemma $4 \varepsilon_{1}$ and $\varepsilon_{2}$ are both positive (see Appendix B).

\section{A.2.2 Follower Considering Technology 1 to Be Interesting}

If $Y \geq Y_{11}^{F}$ the value function of the leader is given by

$$
L_{11}(Y)=\frac{Y D_{11}}{r-\mu}-I
$$

Next we derive the value function of the leader for $Y<Y_{11}^{F}$. The value of the leader equals

$$
L_{11}(Y)=E\left[-I+\int_{t=0}^{\min \left(T, T_{11}^{F}\right)} Y(t) D_{10} \exp (-r t) d t+1_{\left\{T \leq T_{11}^{F}\right\}} \exp (-r T) \Lambda_{12}(Y(T))\right.
$$

Define

$$
\left.\int_{t=\min \left(T, T_{11}^{F}\right)}^{\infty} 1_{\left\{T_{11}^{F}<T\right\}} Y(t) D_{11} \exp (-r t) d t \mid Y(0)=Y\right]
$$

$$
f(Y)=E\left[\int_{t=0}^{\min \left(T, T_{11}^{F}\right)} Y(t) D_{10} \exp (-r t) d t \mid Y(0)=Y\right]
$$

The function $f$ must satisfy the following Bellman equation for $Y<Y_{11}^{\vec{F}}$ :

$$
r f(Y)=Y D_{10}+\lim _{d t \downarrow 0} \frac{1}{d t} E[d f(Y)] .
$$

Itô's lemma gives

$$
E[d f(Y)]=\lambda d t(0-f(Y))+(1-\lambda d t)\left(\frac{\partial f(Y)}{\partial Y} \mu Y d t+\frac{\partial^{2} f(Y)}{\partial Y^{2}} \frac{1}{2} \sigma^{2} Y^{2} d t\right)+o(d t) .
$$

Thus

$$
\mu Y \frac{\partial f(Y)}{\partial Y}+\frac{1}{2} \sigma^{2} Y^{2} \frac{\partial^{2} f(Y)}{\partial Y^{2}}-(r+\lambda) f(Y)+Y D_{10}=0
$$

The solution of this differential equation is given by

$$
f(Y)=v_{1} Y^{\beta_{1}^{*}}+v_{2} Y^{\beta_{2}^{*}}+\frac{Y D_{10}}{r+\lambda-\mu} .
$$


Using the boundary conditions $f(0)=0$ and $f\left(Y_{11}^{F}\right)=0$ the values for the constants are found:

$$
\begin{aligned}
& v_{1}=-\left(Y_{11}^{F}\right)^{1-\beta_{1}^{*}} \frac{D_{10}}{r+\lambda-\mu}, \\
& v_{2}=0 .
\end{aligned}
$$

Next define

$$
\begin{aligned}
g(Y)= & E\left[1_{\left\{T \leq T_{11}^{F}\right\}} \exp (-r T) \Lambda_{12}(Y(T))\right. \\
& \left.+\int_{t=\min \left(T, T_{11}^{F}\right)}^{\infty} 1_{\left\{T_{11}^{F}<T\right\}} Y(t) D_{11} \exp (-r t) d t \mid Y(0)=Y\right] .
\end{aligned}
$$

The function $g$ must satisfy the following Bellman equation

leading to

$$
\begin{aligned}
r g(Y) & =\lim _{d t \downarrow 0} \frac{1}{d t}\left(\lambda d t\left(\Lambda_{12}(Y)-g(Y)\right)\right. \\
& \left.+(1-\lambda d t)\left(\frac{\partial g(Y)}{\partial Y} \mu Y d t+\frac{\partial^{2} g(Y)}{\partial Y^{2}} \frac{1}{2} \sigma^{2} Y^{2} d t+o(d t)\right)\right),
\end{aligned}
$$

$$
\mu Y \frac{\partial g(Y)}{\partial Y}+\frac{1}{2} \sigma^{2} Y^{2} \frac{\partial^{2} g(Y)}{\partial Y^{2}}-(r+\lambda) g(Y)+\lambda \Lambda_{12}(Y)=0
$$

The solution of (69) is given by

$$
g(Y)= \begin{cases}\kappa_{1} Y^{\beta_{1}^{*}}+\kappa_{2} Y^{\beta_{2}^{*}}+B_{12} Y^{\beta_{1}}+\frac{\lambda}{r+\lambda-\mu} \frac{Y D_{10}}{r-\mu} & \text { if } Y<Y_{12}^{F}, \\ \kappa_{3} Y^{\beta_{1}^{*}}+\kappa_{4} Y^{\beta_{2}^{*}}+\frac{\lambda}{r+\lambda-\mu} \frac{Y D_{12}}{r-\mu} & \text { if } Y \geq Y_{12}^{F} .\end{cases}
$$

Due to the boundary condition $g(0)=0$ we know that $\kappa_{2}=0$. The constants $\kappa_{1}, \kappa_{3}$ and $\kappa_{4}$ are found by simultaneously solving the continuity and differentiability condition at $Y=Y_{12}^{F}$ and the boundary condition $g\left(Y_{11}^{F}\right)=\frac{Y_{11}^{F} D_{11}}{r-\mu}$ :

$$
\begin{aligned}
& \kappa_{1}=\kappa_{3}+\varepsilon_{1}, \\
& \kappa_{3}=\left(Y_{11}^{F}\right)^{-\beta_{1}^{*}}\left(\frac{Y_{11}^{F} D_{11}}{r-\mu}-\frac{\lambda Y_{11}^{F} D_{12}}{(r+\lambda-\mu)(r-\mu)}\right)-\left(Y_{11}^{F}\right)^{\beta_{2}^{*}-\beta_{1}^{*}} \varepsilon_{2}, \\
& \kappa_{4}=\varepsilon_{2} .
\end{aligned}
$$


Combining equations (58), (59), (64), and (70) gives equation (29), in which

$$
\begin{aligned}
& \phi_{1}=v_{1}+\kappa_{3}+\varepsilon_{1}, \\
& \phi_{2}=v_{1}+\kappa_{3}, \\
& \phi_{3}=\kappa_{4}=\varepsilon_{2} .
\end{aligned}
$$

Lemma 5 in Appendix B states that $\phi_{1}>0$ and $\phi_{3}>0$.

\section{A.3 Waiting Curve}

The following Bellman equation must hold for the waiting curve

$$
r W(Y)=\lim _{d t \downarrow 0} \frac{1}{d t} E[d W(Y)] .
$$

Itô's lemma gives

$$
\begin{aligned}
E[d W(Y)]= & (1-\lambda d t)\left(\frac{\partial W(Y)}{\partial Y} \mu Y d t+\frac{\partial^{2} W(Y)}{\partial Y^{2}} \frac{1}{2} \sigma^{2} Y^{2} d t\right) \\
& +\lambda d t\left(\Phi_{22}(Y)-W(Y)\right)+o(d t) .
\end{aligned}
$$

Substitution of (78) in (77) gives

$$
r W(Y)=\frac{\partial W(Y)}{\partial Y} \mu Y+\frac{\partial^{2} W(Y)}{\partial Y^{2}} \frac{1}{2} \sigma^{2} Y^{2}+\lambda\left(\Phi_{22}(Y)-W(Y)\right) .
$$

Rewriting gives

$$
\frac{1}{2} \sigma^{2} Y^{2} \frac{\partial^{2} W(Y)}{\partial Y^{2}}+\mu Y \frac{\partial W(Y)}{\partial Y}-(r+\lambda) W(Y)+\lambda \Phi_{22}(Y)=0 .
$$

Using equation (6) and the boundary condition for $Y=0$ and ruling out speculative bubbles,

$$
\begin{aligned}
W(0) & =0, \\
\lim _{Y \rightarrow \infty} \frac{W(Y)}{Y} & =\frac{\lambda D_{22}}{(r+\lambda-\mu)(r-\mu)},
\end{aligned}
$$

gives

$$
W(Y)= \begin{cases}\eta_{1} Y^{\beta_{1}^{*}}+A_{22} Y^{\beta_{1}} & \text { if } Y<Y_{22}^{F} \\ \eta_{2} Y^{\beta_{2}^{*}}+\frac{\lambda Y D_{22}}{(r+\lambda-\mu)(r-\mu)}-\frac{\lambda I}{r+\lambda} & \text { if } Y \geq Y_{22}^{F}\end{cases}
$$


The constants $\eta_{1}$ and $\eta_{2}$ are found by solving the continuity and differentiability conditions for $W$ at $Y=Y_{22}^{F}$ :

$$
\begin{aligned}
& \eta_{1}=\frac{\left(Y_{22}^{F}\right)^{-\beta_{1}^{*}} I\left(r(r-\mu) \beta_{2}^{*}+\left(r-\mu \beta_{1}\right) \lambda \beta_{2}^{*}-(r-\mu)(r+\lambda) \beta_{1}\right)}{(r+\lambda)(r+\lambda-\mu)\left(\beta_{1}-1\right)\left(\beta_{1}^{*}-\beta_{2}^{*}\right)}, \\
& \eta_{2}=\frac{\left(Y_{22}^{F}\right)^{-\beta_{2}^{*}} I\left(r(r-\mu) \beta_{1}^{*}+\left(r-\mu \beta_{1}\right) \lambda \beta_{1}^{*}-(r-\mu)(r+\lambda) \beta_{1}\right)}{(r+\lambda)(r+\lambda-\mu)\left(\beta_{1}-1\right)\left(\beta_{1}^{*}-\beta_{2}^{*}\right)} .
\end{aligned}
$$

A direct result of Lemma 1 is that $\eta_{1}<0$ and $\eta_{2}>0$.

\section{B Lemmas and Proofs}

LEMMA 1

The following two inequalities hold:

$$
\begin{aligned}
& r(r-\mu) \beta_{2}^{*}+\left(r-\mu \beta_{1}\right) \lambda \beta_{2}^{*}-(r-\mu)(r+\lambda) \beta_{1}<0, \\
& r(r-\mu) \beta_{1}^{*}+\left(r-\mu \beta_{1}\right) \lambda \beta_{1}^{*}-(r-\mu)(r+\lambda) \beta_{1}>0 .
\end{aligned}
$$

\section{ProOf OF LEMMA 1}

The assumption $\mu \in(0, r)$ implies that

$$
1 \leq \beta_{1} \leq \frac{r}{\mu}
$$

Equation (86) holds due to equation (88) and the fact that $\beta_{2}^{*}<0$.

We know that $\beta_{1}^{*} \geq \beta_{1}$, where the equality sign only holds for $\sigma \rightarrow \infty$ for which we have $\beta_{1}^{*}=\beta_{1}=1$. Write $\beta_{1}^{*}=\xi \beta_{1}$ and substitute in (87):

$$
\begin{aligned}
& r(r-\mu) \xi \beta_{1}+\left(r-\mu \beta_{1}\right) \lambda \xi \beta_{1}-(r+\lambda)(r-\mu) \beta_{1} \\
= & r(r+\lambda-\mu) \beta_{1}(\xi-1)-\mu \lambda \beta_{1}\left(\xi \beta_{1}-1\right) \\
= & \Xi(\xi) .
\end{aligned}
$$

Then $\Xi(1)=0\left(\xi=1\right.$ implies that $\beta_{1}=\beta_{1}^{*}$ and therefore $\left.\beta_{1}=1\right)$ and

$$
\frac{d \Xi(\xi)}{d \xi}=\beta_{1}\left(r(r+\lambda)-\mu\left(r+\lambda \beta_{1}\right)\right)>0
$$


if and only if

$$
r(r+\lambda)-\mu\left(r+\lambda \beta_{1}\right)>0
$$

Equation (91) holds since

$$
\beta_{1}<\frac{r}{\mu}+\frac{r(r-\mu)}{\mu \lambda}
$$

Therefore equation (87) holds.

\section{LEMMA 2}

The constants $\delta_{1}, \delta_{2}$, and $\delta_{3}$ have the following signs: $\delta_{1}<0, \delta_{2}>0$, and $\delta_{3}>0$.

\section{Proof of Lemma 2}

The signs of $\delta_{2}$ and $\delta_{3}$ follow immediately from equations (47) and (48). Define the following functions:

$$
\begin{aligned}
& Z(Y)=Y^{\beta_{2}^{*}-\beta_{1}^{*}} \frac{\left(1-\beta_{2}^{*}\right) \gamma_{2}}{\beta_{1}^{*}-1}+Y^{-\beta_{1}^{*}} \frac{r I}{\left(\beta_{1}^{*}-1\right)(r+\lambda)}, \\
& V(Y)=Z(Y)+\left(Y_{12}^{F}\right)^{\beta_{2}^{*}-\beta_{1}^{*}} \frac{\beta_{2}^{*} \gamma_{2}}{\beta_{1}^{*}}-\left(Y_{12}^{F}\right)^{1-\beta_{1}^{*}} \frac{D_{21}}{\beta_{1}^{*}(r+\lambda-\mu)} .
\end{aligned}
$$

The first derivative of $Z$ is negative and it can be checked that $V\left(Y_{12}^{F}\right)=0$. Therefore, because $Y_{11}^{F}>Y_{12}^{F}$ it holds that $\delta_{1}=V\left(Y_{11}^{F}\right)<V\left(Y_{12}^{F}\right)=0$.

LEMMA 3

It holds that

$$
\sqrt{2 r \sigma^{2}+\left(\mu-\frac{1}{2} \sigma^{2}\right)^{2}} \geq \mu+\frac{1}{2} \sigma^{2}
$$

Proof of Lemma 3

Squaring both sides of (95) gives

$$
2 r \sigma^{2}+\left(\mu-\frac{1}{2} \sigma^{2}\right)^{2} \geq\left(\mu+\frac{1}{2} \sigma^{2}\right)^{2} .
$$


Rewriting of (96) yields

$$
2(r-\mu) \sigma^{2} \geq 0
$$

Therefore the lemma holds since we assumed that $r>\mu$.

LEMMA 4

The constants $\varepsilon_{1}$ and $\varepsilon_{2}$ are both positive.

\section{Proof OF LEMMA 4}

From equation (56) it directly follows that $\varepsilon_{1}>0$. The lemma holds whenever the following statement is true:

$$
(r-\mu)\left(\beta_{1}-\beta_{1}^{*}\right)+\lambda\left(\beta_{1}-1\right)>0 .
$$

In order to prove that equation (98) holds, define the following function

$$
\Omega(\lambda)=(r-\mu)\left(\beta_{1}-\beta_{1}^{*}(\lambda)\right)+\lambda\left(\beta_{1}-1\right) .
$$

For $\lambda=0$ we have that $\beta_{1}=\beta_{1}^{*}$, so that $\Omega(0)=0$. The second derivative of $\Omega$ is equal to

$$
\frac{\partial^{2} \Omega(\lambda)}{\partial \lambda^{2}}=\frac{(r-\mu) \sigma^{2}}{\left(2(r+\lambda) \sigma^{2}+\left(\mu-\frac{1}{2} \sigma^{2}\right)^{2}\right)^{\frac{3}{2}}}>0 .
$$

Thus the lemma is proved if it holds that

$$
\left.\frac{\partial \Omega(\lambda)}{\partial \lambda}\right|_{\lambda=0} \geq 0
$$

The first derivative of $\Omega$ at $\lambda=0$ equals

Define

$$
\left.\frac{\partial \Omega(\lambda)}{\partial \lambda}\right|_{\lambda=0}=\frac{4 \mu^{2}+4 r \sigma^{2}+\sigma^{4}-\left(4 \mu+2 \sigma^{2}\right) \sqrt{2 r \sigma^{2}+\left(\mu-\frac{1}{2} \sigma^{2}\right)^{2}}}{4 \sigma^{2} \sqrt{2 r \sigma^{2}+\left(\mu-\frac{1}{2} \sigma^{2}\right)^{2}}} .
$$

$$
\eta(r)=4 \mu^{2}+4 r \sigma^{2}+\sigma^{4}-\left(4 \mu+2 \sigma^{2}\right) \sqrt{2 r \sigma^{2}+\left(\mu-\frac{1}{2} \sigma^{2}\right)^{2}} .
$$

Then $\eta(\mu)=0$ and with Lemma 4 we have

$$
\frac{\partial \eta(r)}{\partial r}=\frac{4 \sigma^{2}\left(\sqrt{2 r \sigma^{2}+\left(\mu-\frac{1}{2} \sigma^{2}\right)^{2}}-\left(\mu+\frac{1}{2} \sigma^{2}\right)\right)}{\sqrt{2 r \sigma^{2}+\left(\mu-\frac{1}{2} \sigma^{2}\right)^{2}}} \geq 0 .
$$


This implies that for $r \geq \mu$ equation (101) holds and thereby the lemma is proved.

\section{LEMMA 5}

The constants $\phi_{1}$ and $\phi_{3}$ have the following signs: $\phi_{1}>0$ and $\phi_{3}>0$.

\section{ProOf OF Lemma 5}

We start with $\phi_{3}$. From Lemma 5 we know that $\varepsilon_{2}>0$. Therefore $\phi_{3}>0$.

Define

$$
\begin{aligned}
& E_{1}(Y)=\frac{Y^{1-\beta_{1}^{*}}\left((r-\mu)\left(\beta_{1}-\beta_{2}^{*}\right)+\lambda\left(\beta_{1}-1\right)\right)\left(D_{10}-D_{12}\right)}{(r+\lambda-\mu)(r-\mu)\left(\beta_{1}^{*}-\beta_{2}^{*}\right)}, \\
& E_{2}(Y)=\frac{Y^{1-\beta_{2}^{*}}\left((r-\mu)\left(\beta_{1}-\beta_{1}^{*}\right)+\lambda\left(\beta_{1}-1\right)\right)\left(D_{10}-D_{12}\right)}{(r+\lambda-\mu)(r-\mu)\left(\beta_{1}^{*}-\beta_{2}^{*}\right)} .
\end{aligned}
$$

Then $E_{1}\left(Y_{12}^{F}\right)=\varepsilon_{1}$ and $E_{2}\left(Y_{12}^{F}\right)=\varepsilon_{2}$, further it holds that $E_{1}\left(Y_{11}^{F}\right)<E_{1}\left(Y_{12}^{F}\right)$ and $E_{2}\left(Y_{11}^{F}\right)>E_{2}\left(Y_{12}^{F}\right)$. Further define

$$
K_{3}(Y)=\left(Y_{11}^{F}\right)^{-\beta_{1}^{*}}\left(\frac{Y_{11}^{F} D_{11}}{r-\mu}-\frac{\lambda Y_{11}^{F} D_{12}}{(r+\lambda-\mu)(r-\mu)}\right)-\left(Y_{11}^{F}\right)^{\beta_{2}^{*}-\beta_{1}^{*}} E_{2}(Y),
$$

then it follows after some tedious calculations that

$$
\begin{aligned}
\phi_{1} & =v_{1}+\kappa_{3}+\varepsilon_{1} \\
& =v_{1}+K_{3}\left(Y_{12}^{F}\right)+E_{1}\left(Y_{12}^{F}\right) \\
& >v_{1}+K_{3}\left(Y_{11}^{F}\right)+E_{1}\left(Y_{11}^{F}\right) \\
& =\frac{\left(Y_{11}^{F}\right)^{1-\beta_{1}^{*}}\left(D_{11}-D_{12}\right)}{(r+\lambda-\mu)(r-\mu)}>0 .
\end{aligned}
$$

Thus $\phi_{1}$ is positive.

\section{Proof of Proposition 1}

It is easy to verify that equation (24) does not have a root if $\lambda \geq \lambda_{1}^{*}$. Assertion 2 can be concluded by taking a closer look at equations (24), (20), (47), and (41). The closer $\lambda$ comes to $\lambda_{1}^{*}$ the smaller the negative term in (24) becomes in absolute terms. This implies that $Y_{11}^{F}$ becomes larger. 


\section{Proof of Proposition 2}

From Proposition 1 we know that $Y_{11}^{F}$ does not exists for $\lambda \geq \lambda_{1}^{*}$ and therefore $\phi_{2}$ does not make sense for $\lambda \geq \lambda_{1}^{*}$. First we prove that $\phi_{2} \leq 0$ if equation (30) holds and $\lambda<\lambda_{1}^{*}$. According to (65), (72) and (75) it is sufficient to prove that

$$
\frac{D_{11}}{r-\mu}-\frac{\lambda D_{12}}{(r+\lambda-\mu)(r-\mu)}-\frac{D_{10}}{r+\lambda-\mu} \leq 0 .
$$

Equation (109) holds if

$$
\lambda \leq \frac{(r-\mu)\left(D_{10}-D_{11}\right)}{D_{11}-D_{12}}
$$

Using equation (30) it is not hard to show that

$$
\frac{(r-\mu)\left(D_{10}-D_{11}\right)}{D_{11}-D_{12}}>\lambda_{1}^{*}
$$

Therefore equation (109) holds and $\phi_{2}$ is non-positive.

Let us show that $\phi_{2}$ can be negative when (30) does not hold. Set $\lambda=0$, then $\beta_{1}=\beta_{1}^{*}$ and $\varepsilon_{2}=0$ so that

$$
\phi_{2}=\left(Y_{11}^{F}\right)^{1-\beta_{1}} \frac{D_{11}-D_{10}}{r-\mu}<0 .
$$

Next we argue that $\phi_{2}$ can be positive when equation (30) does not hold. Define the following function

$$
\mathcal{F}_{2}(Y)=Y^{1-\beta_{1}^{*}} \frac{(r-\mu)\left(D_{11}-D_{10}\right)-\lambda\left(D_{12}-D_{11}\right)}{(r+\lambda-\mu)(r-\mu)}-Y^{\beta_{2}^{*}-\beta_{1}^{*}} \varepsilon_{2} .
$$

Thus $\mathcal{F}_{2}\left(Y_{11}^{F}\right)=\phi_{2}$. When equation (30) does not hold, the first term in equation (113) is positive. When $\lambda$ approaches $\lambda_{1}^{*}$ we know from Proposition 1 that $Y_{11}^{F}$ approaches infinity. Taking a closer look at equation (113) we see that the second term goes faster to zero than the first term. Thus for $\lambda$ close enough to $\lambda_{1}^{*}$ we have that $\phi_{2}$ is positive.

\section{Proof of Proposition 3}

This proposition is easily verified by taking a closer look at equations (23), (32), (40), (41), (84), and (85). 


\section{Proof of Proposition 4}

Define the function $\mathcal{L}$ as follows

$$
\mathcal{L}(Y)=L_{11}(Y)-F_{11}(Y)
$$

The functions $L_{11}$ and $F_{11}$ are continuous. Further it holds that $\mathcal{L}(0)=-I$ and $\mathcal{L}\left(Y_{11}^{F}\right)=0$.

Therefore it is sufficient to prove that

$$
\left.\frac{\partial \mathcal{L}(Y)}{\partial Y}\right|_{Y=Y_{11}^{F}}<0 .
$$

Substitution of equations (23) and (29) in (115) gives for $Y \in\left[Y_{12}^{F}, Y_{11}^{F}\right]$ :

$$
\begin{aligned}
\mathcal{L}(Y)= & \left(\phi_{2}-\delta_{2}\right) Y^{\beta_{1}^{*}}+\left(\phi_{3}-\delta_{3}\right) Y^{\beta_{2}^{*}} \\
& +\frac{(r-\mu) D_{10}-\lambda\left(D_{21}-D_{12}\right)}{(r+\lambda-\mu)(r-\mu)} Y-\frac{r I}{r+\lambda} .
\end{aligned}
$$

Thus

$$
\begin{aligned}
\frac{\partial \mathcal{L}(Y)}{\partial Y}= & \beta_{1}^{*}\left(\phi_{2}-\delta_{2}\right) Y^{\beta_{1}^{*}-1}+\beta_{2}^{*}\left(\phi_{3}-\delta_{3}\right) Y^{\beta_{2}^{*}-1} \\
& +\frac{(r-\mu) D_{10}-\lambda\left(D_{21}-D_{12}\right)}{(r+\lambda-\mu)(r-\mu)}
\end{aligned}
$$

From $\mathcal{L}\left(Y_{11}^{F}\right)=0$ we obtain

$$
\begin{aligned}
\left.\frac{Y_{11}^{F}}{\beta_{1}^{*}} \frac{\partial \mathcal{L}(Y)}{\partial Y}\right|_{Y=Y_{11}^{F}}= & \frac{\beta_{2}^{*}-\beta_{1}^{*}}{\beta_{1}^{*}}\left(\phi_{3}-\delta_{3}\right)\left(Y_{11}^{F}\right)^{\beta_{2}^{*}}+\frac{r I}{r+\lambda} \\
& +\frac{1-\beta_{1}^{*}}{\beta_{1}^{*}} \frac{(r-\mu) D_{10}-\lambda\left(D_{21}-D_{12}\right)}{(r+\lambda-\mu)(r-\mu)} Y_{11}^{F} .
\end{aligned}
$$

Subtracting $\frac{1}{\beta_{1}^{*}}$ times equation (24) from equation (118) gives

$$
\begin{aligned}
\left.\frac{Y_{11}^{F}}{\beta_{1}^{*}} \frac{\partial \mathcal{L}(Y)}{\partial Y}\right|_{Y=Y_{11}^{F}}= & \frac{\beta_{2}^{*}-\beta_{1}^{*}}{\beta_{1}^{*}} \phi_{3}\left(Y_{11}^{F}\right)^{\beta_{2}^{*}} \\
& +\frac{1-\beta_{1}^{*}}{\beta_{1}^{*}} \frac{\lambda\left(D_{12}-D_{11}\right)-(r-\mu)\left(D_{11}-D_{10}\right)}{(r+\lambda-\mu)(r-\mu)} Y_{11}^{F}
\end{aligned}
$$

From the proof of Proposition 2 we know that

$$
\lambda \leq \frac{(r-\mu)\left(D_{10}-D_{11}\right)}{D_{11}-D_{12}} .
$$

Equations (119) and (120) together with $\phi_{3}>0$ imply equation (115). 


\section{Proof of Proposition 5}

Define $P(Y)=\operatorname{Pr}\left(T_{S}<T \mid Y(0)=Y\right)$. Then for $Y<S$ the function $P$ must satisfy the following Bellman equation

$$
0=-\lambda P(Y)+\frac{\partial P(Y)}{\partial Y} \mu Y+\frac{\partial^{2} P(Y)}{\partial Y^{2}} \frac{1}{2} \sigma^{2} Y^{2} .
$$

Since $P(0)=0$ and $P(S)=1$ the solution of (121) equals (36).

\section{Proof of Proposition 6}

Taking a closer look at equations (19) and (28) (for $Y$ large) we see that there does exist a crossing point of $L_{12}$ and $F_{12}$ if

$$
\frac{Y D_{10}}{r+\lambda-\mu}+\frac{\lambda}{r+\lambda-\mu} \frac{Y D_{12}}{r-\mu}>\frac{\lambda}{r+\lambda-\mu} \frac{Y D_{21}}{r-\mu} .
$$

Rewriting (122) gives (33).

\section{Proof of Proposition 7}

This proof follows the same lines as the proof of Proposition 6, but then with equations (28) and (32).

\section{REFERENCES}

Dixit, A. K. AND R. S. PindycK (1996). Investment Under Uncertainty, second printing. Princeton University Press, Princeton, New Jersey, United States of America.

FudenberG, D. And J. Tirole (1985). Preemption and rent equalization in the adoption of new technology. The Review of Economic Studies, 52, 383-401.

Göтz, G. (2000). Strategic timing of adoption of new technologies under uncertainty: A note. International Journal of Industrial Organization, 18, 369-379.

Grenadier, S. R. AND A. M. Weiss (1997). Investment in technological innovations: An 
option pricing approach. Journal of Financial Economics, 44, 397-416.

Hendricks, K., A. Weiss, And C. Wilson (1988). The war of attrition in continuous time with complete information. International Economic Review, 29, 663-680.

Hoppe, H. C. (2000). Second-mover advantages in the strategic adoption of new technology under uncertainty. International Journal of Industrial Organization, 18, 315-338.

Huisman, K. J. M. And P. M. Kort (1999a). Effects of strategic interactions on the option value of waiting. CentER Discussion Paper 9992, Tilburg University, CentER, Tilburg, The Netherlands.

Huisman, K. J. M. And P. M. KorT (1999b). Strategic technology investment under uncertainty. CentER Discussion Paper 9918, Tilburg University, CentER, Tilburg, The Netherlands.

Nielsen, M. J. (1999). Competition and irreversible investments. Working Paper, University of Copenhagen, Institute of Economics, Copenhagen, Denmark.

StenbackA, R. And M. M. Tombak (1994). Strategic timing of adoption of new technologies under uncertainty. International Journal of Industrial Organization, 12, 387-411. 